\section{Anicia Peters and}

Heike Winschiers-Theophilus,

Namibia University of Science and Technology
Tuomo Kujala (Ed.),

ACM SIGCHI

\title{
HCI out of Namibia
}

\section{Par}

$\mathrm{n}$ the beginning of 2016, we formed the Windhoek ACM SIGCHI chapter in Namibia. The chapter comprises academics, students, and professionals from across Namibia. Forming a local chapter was a natural step for us, especially at the Namibia University of Science and Technology (NUST, formerly known as Polytechnic of Namibia), but also for other researchers and students, for instance, at the University of Namibia. HCI had already played a central role in almost every computer science student's life in past years, and it was embedded in most of our research clusters. Students were exposed to HCI courses, and research projects have a strong HCI component.

$\mathrm{HCI}$ is embedded in major researchfocus areas of the Computing and Informatics Faculty at NUST, such as the digitization of indigenous knowledge. In collaboration with indigenous rural communities, HCI principles and methods have been reappropriated to local epistemologies. Another strong area of HCI application has been our community and research initiatives with marginalized communities, such as the San (Bushmen) people or unemployed youth in informal settlements. Further interesting work in embedding HCI includes the cyber security, e-participation, big data, and culture research clusters.

In October 2016, one of our research clusters held an International Culture and Computer Science Conference in cooperation with ACM SIGCHI. The conference not only focused on virtual/ augmented reality and multimedia technologies for creative industries and cultural establishments like museums, but it also showcased work in cross-cultural and indigenous cultural designs, such as interactive musical boxes. The conference attracted 108 participants from 13 different countries, including more than 30 local and international students.

The chapter members have also been actively involved in integrating select HCI methods into their hackathons and mobile-applicationdevelopment training. Students have been exposed to personas, evaluations, user testing, informant sessions, paper prototyping, and so on. Students learned principles of the user-centered design approach, even though they were not explicitly explained. An upcoming stream of research relying heavily on HCI is on the issue of gender-based violence (GBV), where very few tech solutions currently exist. This is a sensitive and stigmatized area of research in which traditional methods of data collection fail; thus the methods have to be dynamic. For example, a traditional African hut is used to house an interactive roleplaying application combined with physical props to gather data about GBV. This data in turn informs and provides new directions for a social media campaign against GBV.

AfriCHI '16 was another initiative in which the chapter members were involved in organizing and attending the conference in Nairobi in November 2016. AfriCHI, hosted in cooperation with ACM SIGCHI, was the inaugural Africa Human-Computer Interaction conference that sought to bring $\mathrm{HCI}$ researchers and professionals together across Africa. More than 200 participants attended from 21 different countries, including 13 in Africa. The conference offered workshops, courses, papers, panels, posters, a Design Challenge and an Africa Women in Computing lunch. The conference also hosted the first Summit on Community Networks in Africa by the Internet Society. Remote participants could tune into the conference virtually. The conference was organized by 43 committee members, either based in, from, or interested in Africa under the theme of Kujenga madaraja, kubomoa vizuizi, which means "building bridges, breaking barriers.” The contributions were published in the ACM Digital Library.

(1) Anicia Peters is dean of the Faculty of Computing and Informatics at Namibia University of Science and Technology. She chairs the Windhoek SIGCHI chapter. $\rightarrow$ apetersanust.na

(4) Heike Winschiers-Theophilus is a professor in computer science in the Faculty of Computing and Informatics at Namibia University of Science and Technology. She is the vice chair of the chapter.

$\rightarrow$ hwinschiersanust.na

(4) Tuomo Kujala is ACM SIGCHI vice president for local chapters.

$\rightarrow$ sigchi-vp-chaptersdacm.org 